\title{
Unterscheidet sich das Hausaufgabenverhalten von Schülern aus unterschiedli- chen Klassen? \\ Befunde einer Mehrebenenanalyse im Fach Französisch
}

\section{Inge Schnyder, Alois Niggli, Rico Cathomas und Ulrich Trautwein}

Der Hausaufgabenvergabe wird ein signifikanter Beitrag zur Erklärung von Schulleistung zugesprochen. Bislang ist jedoch weitgehend ungeklärt, wie stark sich unterschiedliche Klassen überhaupt in ihrem mittleren Hausaufgabenverhalten unterscheiden und ob sich Faktoren auf Aggregatsebene (z.B. Kursniveau, Klassengrösse, Kantonszugehörigkeit) in dem Hausaufgabenerledigungsverhalten von Schülerinnen und Schülern niederschlagen. In der vorliegenden Untersuchung mit insgesamt 1312 Achtklässlern aus 73 Klassen mit deutscher Unterrichtssprache aus drei Schweizer Kantonen wurden Mehrebenenanalysen verwendet, um Hausaufgabenmotivation und Hausaufgabenverhalten von Schülerinnen und Schülern vorherzusagen. Die Analysen zeigen, dass Faktoren auf Klassen- und Schulebene in der Tat das häusliche Lernverhalten von Schülern in bedeutsamer Weise beeinflussen. Gleichzeitig wird deutlich, dass eine alleinige Berücksichtigung struktureller Aspekte wie Klassengrösse und Kursniveau nicht ausreichend ist. Die Erforschung der Hausaufgabenqualität wird angemahnt.

\section{Einleitung}

Hausaufgaben gehören zu denjenigen Phänomenen, die - gemessen an ihrer tagtäglichen Bedeutung für Lehrkräfte und Schüler sowie oft auch deren Eltern ein auffallend geringes Forschungsinteresse in der Erziehungswissenschaft und pädagogischen Psychologie genossen haben (vgl. Cooper, 1989; Cooper, Robinson \& Patall, 2006; Trautwein \& Köller, 2003a; Wagner \& Spiel, 2002a). Festmachen lassen sich diese Defizite an der fehlenden Untersuchung von Unterschieden in der Hausaufgabenerledigung zwischen einzelnen Schulklassen. Bis heute blieb empirisch weitgehend ungeklärt, ob sich zwischen einzelnen Klassen überhaupt ein Unterschied in der mittleren Hausaufgabenmotivation und dem mittleren Hausaufgabenengagment feststellen lässt und für welche Hausaufgabenvariablen dieser Unterschied besonders gross oder klein ausfällt. Zudem ist unklar, welche Faktoren auf Klassenebene (z.B. Kursniveau, Hausaufgabenqua- 
lität) bzw. Schulebene (z.B. kulturelles Umfeld, Kantonszugehörigkeit) einen Zusammenhang mit Hausaufgabenmotivation und -verhalten aufweisen.

Die genannten Fragen stehen entsprechend im Mittelpunkt eines vom Schweizerischen Nationalfonds zur Förderung der wissenschaftlichen Forschung geförderten Projekts zur Erforschung der Wirkung von Hausaufgaben in Französisch als Fremdsprache (vgl. Schnyder, Niggli, Cathomas, Trautwein \& Lüdtke, 2006). Der vorliegende Beitrag stellt erste Befunde des Projekts zu zwei wichtigen Fragestellungen vor. (a) Wie stark variieren verschiedene Indikatoren des Hausaufgabenverhaltens und der Hausaufgabenmotivation zwischen unterschiedlichen Klassen? (b) Lassen sich solche Unterschiede in einem stark föderal geprägten Schulsystem wie dem der Schweiz, das zudem eine ausgeprägte Leistungsdifferenzierung aufweist, ggf. durch strukturelle Variablen (Kantonszugehörigkeit, Zweisprachigkeit, Klassengrösse und Kursniveau) erklären?

Im Theorieteil beschreiben wir zunächst die Mehrdimensionalität des Hausaufgabensverhaltens und der Hausaufgabenmotivation, bevor wir auf die Mehrebenenproblematik bei der Erforschung von Hausaufgaben eingehen, die potenzielle Rolle von Strukturmerkmalen beschreiben und die Fragestellung der vorliegenden Arbeit ableiten.

\section{Multidimensionalität des Hausaufgabenverhaltens}

Über einen langen Zeitraum stand die Variable «Hausaufgabenzeit» im Mittelpunkt der Forschung zur Effektivität von Hausaufgaben (vgl. Cooper, 1989). Zwischen der Menge der erteilten Hausaufgaben (als Effekt auf Klassenebene) bzw. dem individuellen Zeiteinsatz für Hausaufgaben (als Effekt auf der Individualebene) auf der einen Seite und dem Leistungszuwachs auf der anderen Seite wurde im Anschluss an die Modelle von Carroll (1963) bzw. Walberg \& Paschal (1995) ein quasi-linearer Zusammenhang vermutet. In jüngerer Zeit wurde die Variable Zeitaufwand zunehmend durch weitere Indikatoren ergänzt (z.B. Muhlenbruck et al., 2000). Trautwein und Köller (2003b) konzipierten das Hausaufgabenverhalten von Schülerinnen und Schülern multidimensional, indem sie zwischen Zeitvariablen und drei zentralen Indikatoren der Selbstregulation (Hausaufgabenengagement, kognitive und metakognitive Lernstrategien) unterschieden. ${ }^{1}$

Der ausserschulische Zeitaufwand umfasst den durchschnittlichen Zeitaufwand für die Hausaufgaben, die durchschnittliche wöchentliche Zusatzlernzeit, beispielsweise für die freiwillige Wiederholung des Stoffes oder die Lösung zusätzlicher Aufgaben sowie die Lernzeit für Klassenarbeiten (Haag \& Mischo, 2002; Trautwein \& Köller, 2003b; Wagner \& Spiel, 2002b). Im Fremdsprachenunterricht lässt sich zusätzlich zwischen dem Zeitaufwand fürs Vokabellernen und für die übrigen Hausaufgaben unterscheiden. Eine Reihe jüngerer Studien, in denen die für die Hausaufgaben aufgewendete Zeit in Beziehung zur Leistung bzw. zur Leistungsentwicklung gesetzt wurde, erbrachten Hinweise darauf, dass die aufgewendete Zeit in keinem oder sogar in einem negativen Zusammenhang mit Schulleistung steht (Muhlenbruck et al., 2000; Trautwein et al., 2002). So be- 
richteten Trautwein und Köller (2003b) für das Fach Mathematik eine signifikante Korrelation von -.23 bzw. - .28 zwischen dem durchschnittlichen Zeitaufwand für Hausaufgaben und der letzten Zeugnisnote bzw. Klassenarbeitsnote. In einer Studie, in der ein Hausaufgabentagebuch zur Erfassung des Zeitaufwands für Hausaufgaben im Lateinunterricht eingesetzt wurde, fanden Haag und Mischo (2002) keinen Zusammenhang zwischen insgesamt aufgewendeter Hausaufgabenzeit und dem Leistungszuwachs. Allerdings wiesen sogenannte «Saisonarbeiter», die nur für die Tage kurz vor und nach Klassenarbeiten einen erhöhten Lernaufwand berichteten, einen signifikant geringeren Leistungszuwachs auf als Mitschüler, die regelmässiger lernten.

Neben dem Zeitaufwand für Hausaufgaben nannten Trautwein und Köller (2003b) das Hausaufgabenengagement als zweite Komponente des Hausaufgabenverhaltens. Das Hausaufgabenengagement, auch als Ressourcenmanagement bezeichnet, wird hierbei mit Hilfe von Aussagen über das typische Hausaufgabenverhalten (z.B. «Ich schreibe die Französisch-Hausaufgaben oft von anderen in der Schule ab»; "Wie viel Prozent der Hausaufgaben versuchst du durchschnittlich ernsthaft zu bearbeiten?») erfasst. Lehrkräfte und Eltern wünschen sich, dass Hausaufgaben ernsthaft bearbeitet werden - nicht aber, dass die Bearbeitung lange Zeit in Anspruch nimmt. Masse des Hausaufgabenengagements zielen direkt auf die Erfassung eines solchen ernsthaften Hausaufgabenverhaltens ab. In der Studie von Trautwein und Köller (2003b) zeigten sich - auch nach Kontrolle von Geschlecht, Schulform, kognitiver Grundfähigkeit und letzter Zeugnisnote - signifikant positive Effekte des Hausaufgabenengagements auf Klassenarbeitsnoten.

Kognitive und metakognitive Lernstrategien sind zwei weitere Facetten des Hausaufgabenverhaltens. Elaborations- und Wiederholungsstrategien stellen typische kognitive Lernstrategien dar, während die Adjustierung der Zielsetzung und Überwachungsprozesse («monitoring») Beispiele für metakognitiveLernstrategien sind (vgl. Artelt, 2000; Lockl \& Schneider, 2003). Die Erfassung von Lernstrategien - insbesondere der metakognitiven Komponenten - ist mit besonderen Problemen behaftet und bislang nicht befriedigend gelöst. So korrelieren Selbstberichte und Verhaltensbeobachtungen nur mässig (Jamieson-Noel \& Winne, 2003). Zudem lässt sich argumentieren, dass viele Lehrkräfte Hausaufgaben vornehmlich zur Wiederholung von bereits Gelerntem («drill»; Cooper, 1989) einsetzen und damit die potentielle Wirksamkeit von Lernstrategien eingeschränkt wird. Die beiden letztgenannten Faktoren mögen erklären, warum kognitive und metakognitive Lernstrategien bislang in Hausaufgabenstudien eher wenig berücksichtigt wurden bzw. wenig prädiktive Kraft hatten (Corno, 2000; De Jong et al., 2000).

Nach dem Modell von Trautwein und Köller (2003b) wird das Hausaufgabenengagement in bedeutsamer Weise von der Hausaufgabenmotivation beeinflusst, die fachspezifisch und im Sinne der Erwartungs-Wert-Theorie von Eccles (1983) durch eine Erwartungskomponente («Kann ich die Hausaufgaben lö- 
sen?») und eine Valenzkomponente («Was habe ich davon, die Hausaufgaben zu bearbeiten») konzeptualisiert wird.

\section{Hausaufgaben als Mehrebenenproblem}

Inhaltlich und unter methodischen Gesichtspunkten stellen Hausaufgaben ein klassisches Mehrebenenproblem dar (Trautwein \& Köller, 2003a). Einerseits ist man an Hausaufgabenerledigungseffekten (als Schülervariable) interessiert. Hierbei wird u.a. die Frage untersucht, ob diejenigen Schülerinnen und Schüler einer Klasse, die ihre Hausaufgaben sorgfältiger erledigen als ihre Klassenkameraden, eine besondere Leistungssteigerung aufweisen. Will man ausschliesslich Hausaufgabeneffekte auf Schülerebene untersuchen, bietet es sich an, alle interessierenden Variablen (z.B. aufgewendete Zeit und Sorgfalt) auf Klassenebene zu zentrieren bzw. zu standardisieren, damit jeweils die unterschiedlichen Verhaltensweisen und Lernzuwächse der verschiedenen Schüler einer Klasse verglichen werden können. Ein solches Vorgehen wurde beispielsweise bei Schnyder et al. (2006) gewählt.

Andererseits ist die Forschung aber auch besonders an der Klärung der Frage interessiert, wie die Hausaufgabenvergabe (als Lehrervariable) die Hausaufgabenmotivation, das Hausaufgabenverhalten sowie die Leistungen der Schulklasse beeinflusst. Will man solche Hausaufgabenvergabeeffekte untersuchen, analysiert man beispielsweise, ob diejenigen Schulklassen, in denen umfangreiche Hausaufgaben erteilt werden, einen höheren Lerngewinn aufweisen als Klassen mit weniger Hausaufgaben (vgl. Trautwein, Köller, Schmitz \& Baumert, 2002). Hierbei wird implizit davon ausgegangen, dass sich das Lehrerhandeln auch signifikant im Verhalten der Schüler niederschlägt. Diese Annahme ist bei dem Zeitaufwand für Hausaufgaben bzw. der Häufigkeit von häuslichem Lernen recht plausibel, wenn auch das Ausmass der direkten Beinflussbarkeit der Schüler unklar ist. Bei anderen Hausaufgabenvariablen wie der Sorgfalt der Erledigung müssen Effekte der Lehrperson nicht zwangsläufig auftreten. So ist es durchaus denkbar, dass das Hausaufgabenverhalten von Schülerinnen und Schülern primär von ihrer eigenen Persönlichkeit, dem Verhalten ihrer Eltern oder von ihren kognitiven Grundfertigkeiten beeinflusst ist, weniger dagegen von einer spezifischen Einflussnahme einer individuellen Lehrkraft.

Die Frage, wie stark sich Schülerinnen und Schüler einer Klasse bzw. Schule in ihrem Hausaufgabenverhalten ähneln, ist von hoher pädagogischer Relevanz. Es ist deshalb bemerkenswert, dass bislang praktisch nur für die Hausaufgabenzeit bzw. -häufigkeit überprüft wurde (vgl. Farrow, Tymms \& Henderson, 1999), wie gross die Unterschiede zwischen einzelnen Schulklassen ausfallen. Ob sich verschiedene Schulklassen auch darin unterscheiden, wie «sorgfältig» sie im Schnitt die Hausaufgaben erledigen bzw. ob sie bestimmte Lernstrategien verwenden, ist dagegen völlig ungeklärt, obschon eine solche Prüfung methodisch wenig aufwändig ist. Wenn es Unterschiede zwischen Klassen gibt, müssten sich in sogenannten Mehrebenenanalysen auf Klassenebene deutliche Unterschiede 
in den Ausprägungen der entsprechenden Variablen finden lassen. Statistisch ausgedrückt sollte sich ein merklicher Anteil der Varianz beispielsweise bei der Hausaufgabenzeit und dem Hausaufgabenengagement auf Klassenebene lokalisieren lassen (vgl. Raudenbush \& Bryk, 2002). Die Analysen werden nur wenig verkompliziert, wenn man nicht nur an Individual- und Klasseneffekten interessiert ist, sondern zusätzlich an Unterschieden zwischen Schulen.

\section{Ableitung der Fragestellung}

Die nachfolgend berichteten Befunde stellen den ersten wichtigen Schritt bei einer umfassenden Analyse der Mehrebenenstruktur der Hausaufgabenvergabe und -erledigung dar. Die Analysen erfolgen dabei in einem zweischrittigen Verfahren. Im ersten Schritt untersuchen wir systematisch, wie sehr sich unterschiedliche Schulklassen in der Hausaufgabenmotivation sowie zentralen Indikatoren des häuslichen Arbeitens unterscheiden. Hierbei sollen für die Hausaufgabenmotivation, die häusliche Arbeitszeit, das Engagement bei Hausaufgaben und die verwendeten Lernstrategien im Fach Französisch die Varianzanteile von Schülern, Schulklassen und Schulen bestimmt werden. Wir gehen dabei davon aus, dass für die untersuchten Variablen bedeutsame Varianzanteile auf Klassen- bzw. Schulebene gefunden werden.

Dass solche Unterschiede zwischen Klassen oder Schulen auftreten, ist durchaus keine triviale Vorhersage. Bei einer Reihe von Variablen, die als wichtige Erfolgskriterien pädagogischen Handelns betrachtet werden, konnten nur geringe Varianzanteile auf Klassenebene- oder Schulebene beobachtet werden. So fand sich bei Anderman (2002) für Variablen wie Selbstwertgefühl, Depression, Zugehörigkeitsgefühl zur Schule und soziale Eingebundenheit teilweise deutlich mehr als 92\% der Varianz auf Individualebene. Lediglich für den Notendurchschnitt berichtete Anderman einen Varianzanteil von 10 Prozent auf Schulebene. Ähnlich fanden Stanat und Kunter (2001) für eine Reihe von Indikatoren des sozialen Lernens eine sehr geringe Varianzerklärung durch die Schulzugehörigkeit. Auch bei Variablen, die im Bildungsauftrag von Schule stärker verankert sind, zeigt sich oft nur ein geringer Varianzanteil auf Klassen- oder Schulebene. In Hinblick auf wissenschaftspropädeutische Kenntnisse und epistemologische Überzeugungen berichteten Trautwein und Lüdtke (2004) für rund ein Dutzend Variablen Varianzanteile auf Schulebene, die sich mit wenigen Ausnahmen nahe Null bewegten. Dies weist darauf hin, dass die Ausprägung dieser Kriteriumsvariablen stärker von individuellen Voraussetzungen als durch die Beschulung bestimmt ist bzw. die Beschulung in allen Schulen ähnlich erfolgreich verlief.

Zudem soll in einem zweiten Schritt überprüft werden, ob sich solche Unterschiede - wenn sie denn nachgewiesen werden können - durch strukturelle Prädiktoren erklären lassen. Insgesamt vier potenziell relevante Merkmale auf Aggregatebene werden berücksichtigt. Erstens gilt unser Interesse möglichen 
Unterschieden zwischen den Kantonen, die sowohl auf gewachsene kulturelle Unterschiede als auch auf spezifische Merkmale des jeweiligen Schulsystems zurückgeführt werden könnten. Zweitens untersuchen wir, ob die Zweisprachigkeit einer Stadt eine spezifische Opportunitätsstrukturen für den Erwerb der französischen Sprache darstellt. Drittens überprüfen wir, ob - wie von verschiedener Seite gelegentlich impliziert - ein höheres Kursniveau bzw. ein höherer Bildungsgang mit einem stärkeren Hausaufgabenengagement einhergeht. Viertens soll schliesslich der Zusammenhang von Klassengrösse und Hausaufgabenverhalten untersucht werden: Wird in grossen Klassen ggf. nicht bewältigter Unterrichtsstoff in Form von Hausaufgaben vermittelt, was sich in besonders langen Hausaufgabenbearbeitungszeiten manifestieren sollte?

\section{Methode}

\section{Stichprobe}

Es nahmen insgesamt 1312 Achtklässler (51.4\% weiblich) aus 73 Schulklassen aus 22 Schulen in drei Schweizer Kantonen teil. Die Stichprobe ist Teil einer grösseren Untersuchung zur Wirkung von Hausaufgaben, die an der Pädagogischen Hochschule Freiburg/Fribourg durchgeführt wird. Insgesamt beteiligten sich 108 Klassen an zumindest einem der Messzeitpunkte der längsschnittlich angelegten Untersuchung. Für die vorliegende Untersuchung wurden diejenigen Klassen ausgeschlossen, (1) deren Lehrkräfte an einer Schulung zur Verbesserung der Hausaufgaben teilnahmen, die im Rahmen der grösseren Studie durchgeführt wird oder (2) von denen die entsprechenden Schülerfragebögen nicht vorlagen. Zudem wurde eine Klasse ausgeschlossen, die ausschliesslich von Schülern mit Förderbedarf besucht wird.

Die Studie wird in deutschsprachigen Schulen in drei Schweizer Kantonen durchgeführt. In den deutschsprachigen Teilen der Kantone Freiburg und Wallis ist dabei praktisch von einer Vollerhebung auszugehen. Von 26 in Frage kommenden Schulen beteiligten sich 23 an der Studie (wovon wiederum 18 auch in der vorliegenden Teilstichprobe Berücksichtigung fanden). Dagegen liegt in Luzern ein geringerer Abdeckungsgrad vor, was auf organisatorisch-forschungspraktische Gründe zurück geht. In den teilnehmenden Schulen konnten in der Regel alle Parallelklassen für die Teilnahme an der Studie gewonnen werden. Die Untersuchung fand in den ersten Monaten des Schuljahrs 2003/2004 in regulären Unterrichtsstunden statt. Die Administration der Instrumente wurde von den Lehrkräften vor Ort übernommen, die eine exakte schriftliche Einweisung erhalten hatten.

Instrumente

Hausaufgabenzeit

Die Variablen zur Erfassung der Hausaufgabenzeit, der durchschnittlichen Zu- 
satzlernzeit pro Woche sowie der Lernzeit vor Klassenarbeiten (vgl. Tabelle 1) wurden in Anlehnung an Trautwein und Köller (2003b) konzipiert. Bei der Hausaufgabenzeit und der durchschnittlichen Lernzeit wurden freie Antwortfelder vorgegeben und um Angaben in Minuten gebeten. Die Angaben zur wöchentlichen Zusatzlernzeit wurden anschliessend in Blöcken zu 30 Minuten zusammengefasst $(1=$ bis 30 Minuten, $2=31$ bis 60 Minuten, 3 = 61-90 Minuten, $4=91-120$ Minuten, 5 = mehr als 120 Minuten). Die Angabe zur Lernzeit vor Klassenarbeiten erfolgte in ganzen Stunden auf einer vorgegebenen achtstufige Antwortskala, deren Endanker die Antwortmöglichkeiten «gar nicht» und «mehr als sechs Stunden» bildeten. Zudem wurde im freien Antwortformat nach der Häufigkeit des Vokabellernens pro Woche sowie der dafür jeweils aufgewendeten Zeit (in Minuten) gefragt.

\section{Hausaufgabenengagement}

Während die Hausaufgabenzeitvariablen eine quantitative Messung des Hausaufgabenverhaltens ermöglichen, drückt sich in den Indikatoren des engagierten Hausaufgabenverhaltens die qualitative Komponente aus (vgl. Tabelle 1). Die Sorgfalt der Schüler bei der Hausaufgabenerledigung wurde über eine Skala mit sechs Items erhoben, die sich an die Arbeit von Trautwein und Köller (2003b) anlehnte. Unregelmässiges Lernverhalten im Sinne der «Saisonarbeiter» bei Haag und Mischo (2002) wurde mit vier Items erfasst, die Persistenz bei schwierigen Aufgaben mit zwei Items. Alle gebildeten Skalen erwiesen sich als hinreichend internal konsistent.

\section{Lernstrategien}

Zur Erfassung kognitiver Lernstrategien wurde ein Subset von Items aus dem «Strategy Inventory for Language Learning» (SILL; Oxford, 1990) vorgegeben. Die insgesamt 20 ausgewählten Items entstammten den Bereichen extracurricularer Lernaktivitäten, kognitive Lernaktivitäten, analytische Strategien und Kooperationsstrategien. Faktorenanalytisch liessen sich drei inhaltlich gut nachvollziehbare Skalen bilden (vgl. Tabelle 1). Die Skala Aktive Verwendung umfasst Items, die die konkrete Nutzung der französischen Sprache im Alltag thematisieren. In der Skala Elaboration verschmelzen Items aus den kognitiven und analytischen Subskalen des SILL. Die Skala Kommunikation umfasst die Items der Skala Kooperationsstrategien. Um zudem Elemente von metakognitiven Lernstrategien zu erfassen, wurden zwei Items verwendet, die den Prozess der Lernsteuerung beschreiben ("Monitoring «). Diese Items thematisieren das Ausmass, in dem Schüler aufgrund einer subjektiven Ist-Soll-Analyse des Lernstands entscheiden, selbständig Stoff nachzuarbeiten. Die internen Konsistenzen der Skalen lagen - mit Ausnahme der Skala Kommunikation - im befriedigenden Bereich.

\section{Hausaufgabenmotivation}

Die Erwartngskomponente (5 Items) sowie die Valenzkomponente (4 Items) 
wurden in Anlehnung an die Arbeit von Trautwein und Köller (2003b) operationalisiert. Die beiden Skalen erwiesen sich als hinreichend internal konsistent.

\section{Tabelle 1. Zentrale Indikatoren des Hausaufgabenverhaltens - Items, Mittelwerte $(M)$, Standardabweichungen (SD) und interne Konsistenzen (Cronbachs $\alpha$ )}

\begin{tabular}{|c|c|c|c|c|c|}
\hline Skala/Item & Beispiel/Wortlaut & Items & $\mathrm{M}$ & SD & $\alpha$ \\
\hline \multicolumn{6}{|l|}{ Zeitvariablen } \\
\hline $\begin{array}{l}\text { Hausauf- } \\
\text { gabenzeit }\end{array}$ & $\begin{array}{l}\text { Wenn ihr Hausaufgaben in Französisch aufbekommt, } \\
\text { wie viele Minuten brauchst du dann durchschnittlich, } \\
\text { um diese Französisch-Hausaufgaben zu erledigen } \\
\text { (ohne Wörter lernen)? }\end{array}$ & 1 & 17.93 & 11.00 & $\mathrm{n} / \mathrm{a}$ \\
\hline $\begin{array}{l}\text { Durchschnitt- } \\
\text { liche Zusatz- } \\
\text { lernzeit }\end{array}$ & $\begin{array}{l}\text { Denke bitte an eine normale Woche: Wie viele Minu- } \\
\text { ten lernst du in einer normalen Woche für Französisch } \\
\text { zusätzlich zu den Hausaufgaben und dem Wörterler- } \\
\text { nen (z.B. Stoff wiederholen; freiwillig Aufgaben lösen; } \\
\text { Stoff vorbereiten)? (Item rekodiert, siehe Fliesstext) }\end{array}$ & 1 & $2.09 \mathrm{a}$ & 1.42 & $\mathrm{n} / \mathrm{a}$ \\
\hline $\begin{array}{l}\text { Lernzeit vor } \\
\text { Klassen- } \\
\text { arbeiten }\end{array}$ & $\begin{array}{l}\text { Denke bitte an eine Woche, in der eine Klassenar- } \\
\text { beit/benotete Prüfung geschrieben wird: Wie viel } \\
\text { Stunden lernst du in einer solchen Woche für Franzö- } \\
\text { sich zusätzlich zu den Hausaufgaben? }\end{array}$ & 1 & 2.94 & 1.23 & $\mathrm{n} / \mathrm{a}$ \\
\hline $\begin{array}{l}\text { Häufigkeit } \\
\text { Vokabellernen }\end{array}$ & $\begin{array}{l}\text { An wie vielen Tagen der Woche lernst du durchsch- } \\
\text { nittlich Französisch-Wörter? }\end{array}$ & 1 & 2.92 & 1.50 & $\mathrm{n} / \mathrm{a}$ \\
\hline $\begin{array}{l}\text { Durchschnitt- } \\
\text { liche Zeit für } \\
\text { Vokabellernen } \\
\text { Engagement }\end{array}$ & $\begin{array}{l}\text { Wenn du an einem Tag Französisch-Wörter lernst: } \\
\text { Wie viel Zeit verwendest du dafür durchschnittlich? } \\
\text { (Angabe in Minuten) }\end{array}$ & 1 & 24.50 & 17.47 & $\mathrm{n} / \mathrm{a}$ \\
\hline Sorgfalt & $\begin{array}{l}\text { Ich versuche immer, meine Französisch-Hausaufga- } \\
\text { ben vollständig zu erledigen. }\end{array}$ & 6 & 3.19 & 0.55 & .74 \\
\hline «Saisonarbeiter» & $\begin{array}{l}\text { Bei den Französisch-Hausaufgaben strenge ich mich } \\
\text { eigentlich erst kurz vor Klassenarbeiten wirklich an. }\end{array}$ & 4 & 2.32 & 0.65 & .72 \\
\hline $\begin{array}{l}\text { Persistenz } \\
\text { Aufgaben }\end{array}$ & $\begin{array}{l}\text { Auch bei schwierigen Aufgaben gebe ich nicht schnell } \\
\text { auf. }\end{array}$ & 3 & 2.70 & 0.62 & .60 \\
\hline \multicolumn{6}{|l|}{ Lernstrategien } \\
\hline $\begin{array}{l}\text { Aktive Verwen- } \\
\text { dung }\end{array}$ & $\begin{array}{l}\text { Ich versuche, Französisch zu sprechen, wann immer } \\
\text { sich dafür eine Gelegenheit bietet. }\end{array}$ & 6 & 1.97 & 0.67 & .79 \\
\hline Elaboration & $\begin{array}{l}\text { Wenn ich ein Wort nicht kenne, zerlege ich es oft in } \\
\text { einzelne Teile, die ich verstehe. }\end{array}$ & 6 & 2.42 & 0.56 & .69 \\
\hline $\begin{array}{l}\text { Kommunika- } \\
\text { tion }\end{array}$ & $\begin{array}{l}\text { Ich spreche mit meinen Mitschülern über Aufgaben, } \\
\text { die ich nicht verstanden habe. }\end{array}$ & 3 & 2.54 & 0.67 & .58 \\
\hline Monitoring & $\begin{array}{l}\text { Wenn ich in Französisch etwas nicht verstehe, lese ich } \\
\text { das zu Hause nach. }\end{array}$ & 2 & 2.56 & 0.76 & .71 \\
\hline \multicolumn{6}{|l|}{ Motivation } \\
\hline $\begin{array}{l}\text { Erwartungs- } \\
\text { komponente }\end{array}$ & $\begin{array}{l}\text { Wenn ich möchte, finde ich immer einen Weg, die } \\
\text { Französisch-Hausaufgaben korrekt zu lösen. }\end{array}$ & 5 & 3.20 & 0.51 & .68 \\
\hline $\begin{array}{l}\text { Valenz- } \\
\text { komponente }\end{array}$ & $\begin{array}{l}\text { Die Französischhausaufgaben bringen mir nichts. } \\
\text { (umgepolt) }\end{array}$ & 4 & 3.09 & 0.59 & .75 \\
\hline
\end{tabular}

a Die Angabe des Mittelwerts bezieht sich auf das rekodierte Item, bei der die 2 der Angabe «31 bis 60 Minuten» entspricht. 


\section{Strukturelle Variablen}

Als strukturelle Variablen auf Schulebene wurde zum einen die Kantonszugehörigkeit in Form von zwei Dummy-Variablen, zum anderen eine etwaige Zweisprachigkeit am Standort der jeweiligen Schule berücksichtigt. Das Kursniveau wurde zweistufig konzeptualisiert, wobei einerseits Schüler mit Zugehörigkeit zum Niveau II bzw. zur Realschule sowie andererseits jene aus dem Niveau I und der Sekundarschule/dem Progymnasium zusammengefasst wurden. Die Variable zur Klassengrösse wurde aufgrund von Angaben der Lehrkräfte über die Zahl der Schüler pro Klasse gebildet.

\section{Statistisches Vorgehen}

Im Mittelpunkt der vorliegenden Untersuchung steht die Frage, welcher Anteil der Varianz im Hausaufgabenverhalten der Schüler durch Effekte auf Klassenund Schulebene erklärt werden kann. Es handelt sich damit um eine Fragestellung, in der die hierarchische Gliederung der Daten (Schüler sind innerhalb von Schulklassen und diese wiederum innerhalb von Schulen "genestet») eine zentrale Rolle spielt. Bei der Analyse solcher hierarchisch geschachtelter Datensätze hat sich die Verwendung spezialisierter Programme durchgesetzt. Bis auf die deskriptiven Analysen wurden alle im Folgenden berichteten Befunde mit dem Computerprogramm HLM 5 (Raudenbush, Bryk, Cheong \& Congdon, 2000) durchgeführt. Dieses Vorgehen hat zwei Vorteile: (1) Mit Hilfe des Programms HLM lässt sich auf einfache Art und Weise ermitteln, welcher Anteil der Varianz in dem gegebenen Datensatz auf Individual-, Klassen und Schulebene lokalisiert ist. Dies ist in der vorliegenden Arbeit eine zentrale Information. Je weniger Varianz auf Individualebene liegt, desto mehr unterscheiden sich einzelne Klassen oder Schulen voneinander. (2) Zudem lässt sich überprüfen, ob Prädiktoren auf der jeweiligen Aggregatebene einen signifikanten Anteil der Varianz aufklären können. Hier gibt HLM unstandardisierte Regressionsgewichte aus, die analog der Regressionsgewichte aus multiplen Regressionsanalysen interpretiert werden können. Allerdings berechnet das Programm HLM einen Standardfehler, bei dem die hierarchische Struktur der Daten berücksichtigt wird. Dadurch sinkt die Gefahr von Alphafehlern (ein Prädiktor wird fälschlicher Weise als signifikant erachtet). Eine umfassende, gut lesbare Beschreibung von Mehrebenenanalysen findet sich bei Hox (2002). Im vorliegenden Fall wurden alle Hausaufgabenvariablen z-standardisiert, bevor die HLM-Analysen durchgeführt wurden. Dadurch ist eine einfache Interpretation der Regressionsgewichte der Prädiktoren sowie ein Vergleich über die unterschiedlichen abhängigen Variablen möglich. 


\section{Ergebnisse}

\section{Deskriptive Analysen}

Mittelwerte und Standardabweichungen für die Hausaufgabenvariablen sind in Tabelle 1 ausgewiesen. Wie sich erkennen lässt, benötigten die Schüler nach eigenen Angaben im Durchschnitt 17.93 Minuten $(S D=11.00)$ für die Erledigung der Französisch-Hausaufgaben. Für das Lernen von Vokabeln, das bei der befragten Schülerschaft im Mittel drei Mal pro Woche stattfand, wurden im Durchschnitt rund 25 Minuten aufgewendet. Die durchschnittliche Zusatzlernzeit (z.B. Stoff wiederholen, freiwillig Aufgaben lösen, Stoff vorbereiten) betrug im Durchschnitt rund eine Stunde pro Woche. In der Woche vor einer Klassenarbeit wurde im Durchschnitt drei Stunden zusätzlich gelernt.

Insgesamt gaben die Schülerinnen und Schüler ein eher hohes Hausaufgabenengagement an. Der Mittelwert für die Skala Hausaufgabensorgfalt lag mit $M$ $=3.19(S D=0.55)$ ebenso über dem theoretischen Skalenmittel wie derjenige für die Hausaufgabenpersistenz $(M=2.70, S D=0.62)$. Items der SaisonarbeiterSkala wurden dagegen als weniger zutreffend empfunden. Hinsichtlich der Nutzung von Lernstrategien fand sich der niedrigste Mittelwert bei der Skala zur aktiven Verwendung der Sprache. Die Skalen Elaboration, Kommunikation und Monitoring lagen dagegen in der Nähe des theoretischen Skalenmittelwerts.

\section{Vorhersage von Zeitaufwand, Engagement und Verwendung von Lernstrategien}

Wie ähnlich fällt die Hausaufgabenmotivation und Hausaufgabenerledigung innerhalb von Schulklassen aus? Wie gross sind die Unterschiede zwischen Klassen aus unterschiedlichen Kantonen bzw. Niveaustufen? Zur Beantwortung dieser Fragen wurden Mehrebenenalysen im Programmpaket HLM 5 (Raudenbush et al., 2000) durchgeführt. Für sämtliche Indikatoren des Hausaufgabenverhaltens wurden zwei Modelle spezifiziert, wobei in einem ersten Modell, dem sogenannten Nullmodell, die Varianzverteilung zwischen Schüler-, Klassen- und Schulebene ermittelt wurde und im zweiten Schritt auf Klassen- bzw. Schulebene strukturelle Merkmale (Klassengrösse, Leistungsniveau, Kanton, Zweisprachigkeit) einbezogen wurden. Die Befunde sind den Tabellen 2 (Zeitvariablen) und 3 (Engagement, Lernstrategieren, Motivation) zu entnehmen.

In Bezug auf die Hausaufgabenzeit als abhängiger Variable zeigte sich zunächst im Nullmodell, dass rund 88 Prozent der Gesamtvarianz auf Ebene der Schülerinnen und Schüler lokalisiert waren (siehe die drei Zeilen zur Varianzaufteilung). In anderen Worten: Auch Schülerinnen und Schüler aus ein- und derselben Klasse berichteten einen sehr unterschiedlichen Zeitaufwand für die Französisch-Hausaufgaben. Rund 11 Prozent der Varianz lagen zwischen den Schulklassen, während auf der Ebene von Schulen nur ein Prozent der Gesamtvarianz lokalisiert war. 
Tabelle 2. Mehrebenenanalysen mit den Zeitvariablen zur Ermittlung der Varianzverteilung zwischen Individual-, Klassen- und Schulebene sowie zur Überprüfung der prädiktiven Kraft struktureller Variablen. Unstandardisierte Regressionskoeffizienten

\begin{tabular}{|lccccc|}
\hline & $\begin{array}{c}\text { Zeit je } \\
\text { Hausauf- } \\
\text { gabe }\end{array}$ & $\begin{array}{c}\text { Extrazeit/ } \\
\text { Woche }\end{array}$ & $\begin{array}{c}\text { Zeit vor } \\
\text { Klausuren }\end{array}$ & $\begin{array}{c}\text { Vokabeln: } \\
\text { Tage }\end{array}$ & $\begin{array}{c}\text { Vokabeln: } \\
\text { Zeitauf- } \\
\text { wand }\end{array}$ \\
\hline $\begin{array}{l}\text { Schulebene } \\
\text { Wallis }\end{array}$ & 0.12 & $0.39^{* *}$ & $0.25^{*}$ & $0.70^{* * *}$ & -0.04 \\
Luzern & & & & & \\
Zweisprachigkeit & -0.05 & -0.14 & 0.19 & 0.05 & 0.07 \\
Klassenebene & $-0.40^{* * *}$ & 0.00 & -0.30 & $-0.24^{* *}$ & -0.43 \\
Klassengröße & & & & & \\
Kursniveau & 0.01 & -0.01 & $0.02^{*}$ & 0.00 & 0.02 \\
\hline $\begin{array}{l}\text { Varianzverteilung (Nullmodell) } \\
\text { Level-3 }\end{array}$ & $-0.29^{*}$ & -0.12 & -0.07 & 0.11 & -0.21 \\
Level-2 & 0.01 & 0.05 & 0.04 & 0.17 & 0.04 \\
Level-1 & 0.11 & 0.14 & 0.01 & 0.09 & 0.09 \\
Varianzaufklärung durch Prädiktoren & 0.88 & 0.81 & 0.95 & 0.74 & 0.87 \\
Level-3 & 0.99 & 0.93 & 0.67 & 0.96 & 0.09 \\
Level-2 & 0.24 & 0.06 & 0.00 a & 0.00 a & 0.09 \\
\hline
\end{tabular}

Anmerkungen. Alle Analysen wurden mit HLM 5 (Raudenbush et al., 2000) durchgeführt. a Schätzung der Varianzaufklärung ergab einen geringfügig negativen Wert (vgl. Fließtext).

${ }^{* * *} p<.001 ;{ }^{* *} p<.01 ;{ }^{*} p<.05$.

bReferenzgruppe: Freiburg

Das bedeutet, dass sich einzelne Klassen bedeutsam in der mittleren Hausaufgabenzeit unterscheiden, zwischen Schulen dagegen nur eine geringe systematische Variation auftrat. In einem zweiten Schritt wurden die fünf Aggregatvariablen berücksichtigt. Auf Ebene der Schulen zeigte sich, dass Schüler von Schulen in zweisprachigen Gebieten deutlich weniger Zeitaufwand für die Hausaufgaben berichteten als die übrigen untersuchten Schüler. Zudem war auf Klassenebene ein höheres Kursniveau mit einem niedrigeren Lernaufwand verbunden. Die berükksichtigten Prädiktoren erklärten die (sehr geringen) Unterschiede auf Schulebene fast vollständig, während auf Klassenebene lediglich ein Viertel der Varianz erklärt wurde (siehe die Zeilen zur Varianzaufklärung). ${ }^{2}$

Betrachtet man die übrigen Zeitvariablen, so sind zwei Aspekte besonders auffällig. Zum einen variierten die Varianzanteile, die auf Schülerebene lagen, recht beträchtlich (zwischen rund 74 und 95 Prozent). Dass die Varianzanteile für die Klassen- und Schulebene bei der Häufigkeit des Vokabellernens am höchsten sind, ist durchaus plausibel, da sich hier direkt die Häufigkeit der Hau- 
saufgabenvergabe niederschlagen kann. Zum anderen fand sich bei drei dieser vier Zeitvariablen auf Schulebene ein signifikanter und durchaus auffälliger Erklärungsbeitrag der Kantonszugehörigkeit: Schüler aus dem Kanton Wallis gaben im Vergleich zu der Referenzkategorie (Schüler aus Freiburg) an, durchschnittlich mehr Zeit pro Woche zusätzlich zu den Hausaufgaben für Französisch zu lernen, mehr Zeit vor Klassenarbeiten zu investieren und an mehr Tagen Vokabeln zu lernen. Hinsichtlich der übrigen Prädiktorvariablen gab es nur zwei signifikante Befunde, die einerseits die Mehrsprachigkeit des Schulorts (in mehrsprachigen Orten wurden Vokabeln weniger häufig gelernt) und andererseits die Klassengrösse (in grösseren Klassen wurde vor Klassenarbeiten mehr Zeit für das Lernen aufgewendet) betrafen.

Die Analysen zu den Indikatoren des engagierten Hausaufgabenverhaltens wurden in identischer Weise durchgeführt (vgl. Tabelle 3). In Hinblick auf die Sorgfalt der Hausaufgabenerledigung waren knapp 9 Prozent der Varianz auf Klassenebene und rund 3 Prozent auf Schulebene lokalisiert. Keiner der berücksichtigten strukturellen Prädiktoren war signifikant. Letzteres gilt auch für die Skala Persistenz, bei der jedoch bereits die einfache Varianzzerlegung deutlich macht, dass hier insbesondere auf Schülerebene nach Quellen der Varianz zu suchen ist, da nur rund 3 Prozent der Varianz nicht auf die Individualebene entfielen. Komplexer stellt sich das Bild in Hinblick auf die Saisonarbeiter-Skala dar, bei der fast neun Prozent der Varianz auf Klassen- bzw. Schulebene lokalisiert war. Auffällig ist, dass mit dem Kanton Wallis und der Zweisprachigkeit des Schulorts genau dieselben Prädiktoren signifikant waren wie bei der Häufigkeit des Wörterlernens. Dies ist angesichts der inhaltlichen Nähe dieser beiden Prädiktoren inhaltlich gut nachvollziehbar.

In einem nächsten Schritt sollen die Lernstrategien in Französisch betrachtet werden. Am auffälligsten sind die Befunde zur Skala «aktive Nutzung der französischen Sprache», bei der immerhin 15 Prozent der Varianz auf Schulebene lokalisiert waren. Bis auf das Kursniveau waren in Bezug auf diese Skala alle Prädiktoren signifikant. Die Varianzverteilung für die übrigen drei Lernstrategien fiel recht ähnlich aus (zwischen 90 und 93\% der Varianz auf Individualebene). Bis auf die Skala Monitoring, bei der das Kursniveau (Schüler in den leistungsstärkeren Kursen geben mehr Monitoring an) und die Zugehörigkeit zum Kanton Wallis (Schüler im Wallis wiesen nach Kontrolle der anderen Prädiktoren einen höheren Wert auf) signifikante Regressionsgewichte hatten, verfehlten alle anderen Prädiktorvariablen das Signifikanzniveau.

Zuletzt soll noch die Hausaufgabenmotivation betrachtet werden. Insbesondere für die Valenzkomponente zeigten sich grosse Unterschiede zwischen den Klassen. Immerhing 14 Prozent der Gesamtvarianz waren auf Klassenebene lokalisiert. In Hinblick auf die Erwartungskomponente fiel der Anteil an Varianz, der nicht auf die Individualebene, mit insgesamt sieben Prozent geringer aus. Die Effekte der Prädiktorvariablen verfehlten allesamt das Signifikanzniveau. 
Tabelle 3. Mehrebenenanalysen zur Ermittlung der Varianzverteilung im Hausaufgabenverhalten und der Hausaufgabenmotivation zwischen Individual-, Klassen- und Schulebene sowie zur Überprüfung der prädiktiven Kraft struktureller Variablen. Unstandardisierte Regressionskoeffizienten

\begin{tabular}{|c|c|c|c|c|c|c|c|c|c|}
\hline & \multicolumn{3}{|c|}{ Engagement } & \multicolumn{4}{|c|}{ Lernstrategien } & \multicolumn{2}{|c|}{ Motivation } \\
\hline & Sorgfalt & $\begin{array}{l}\text { Saison- } \\
\text { arbeiter }\end{array}$ & $\begin{array}{c}\text { Persis- } \\
\text { tenz }\end{array}$ & $\begin{array}{l}\text { Aktive } \\
\text { Nut- } \\
\text { zung }\end{array}$ & $\begin{array}{l}\text { Elabo- } \\
\text { ration }\end{array}$ & $\begin{array}{c}\text { Kommu } \\
\text {-nika- } \\
\text { tion }\end{array}$ & $\begin{array}{l}\text { Moni- } \\
\text { toring }\end{array}$ & $\begin{array}{c}\text { Erwar- } \\
\text { tung }\end{array}$ & Valenz \\
\hline \multicolumn{10}{|l|}{ Schulebene } \\
\hline Wallis ${ }^{b}$ & 0.07 & $-0.24^{* *}$ & 0.00 & $-0.16^{*}$ & 0.18 & 0.11 & $0.29^{*}$ & 0.01 & 0.04 \\
\hline Luzern $^{b}$ & 0.14 & 0.01 & -0.13 & $-0.41^{* *}$ & 0.05 & 0.04 & -0.06 & -0.17 & -0.23 \\
\hline Zweisprachigkeit & -0.28 & $0.17^{* *}$ & -0.02 & $1.27^{* * *}$ & -0.22 & -0.18 & 0.02 & 0.05 & -0.27 \\
\hline \multicolumn{10}{|l|}{ Klassenebene } \\
\hline Klassengrösse & 0.01 & -0.01 & 0.01 & $0.02^{*}$ & 0.02 & 0.00 & 0.00 & 0.01 & 0.01 \\
\hline Kursniveau & 0.06 & 0.11 & -0.10 & -0.10 & -0.07 & -0.03 & $0.25^{*}$ & 0.08 & -0.02 \\
\hline \multicolumn{10}{|c|}{ Varianzverteilung (Nullmodell) } \\
\hline Level-3 & 0.03 & 0.01 & 0.02 & 0.15 & 0.02 & 0.05 & 0.05 & 0.02 & 0.00 \\
\hline Level-2 & 0.09 & 0.07 & 0.01 & 0.02 & 0.04 & 0.04 & 0.05 & 0.05 & 0.14 \\
\hline Level-1 & 0.88 & 0.91 & 0.97 & 0.83 & 0.93 & 0.91 & 0.90 & 0.93 & 0.86 \\
\hline \multicolumn{10}{|c|}{ Varianzaufklärung durch Prädiktoren } \\
\hline Level-3 & 0.35 & 1.00 & 0.30 & 1.00 & 0.87 & 0.13 & 0.39 & 0.35 & 0.25 \\
\hline Level-2 & 0.02 & 0.13 & $0.00^{\mathrm{a}}$ & $0.00^{\mathrm{a}}$ & 0.08 & 0.01 & 0.34 & 0.10 & 0.09 \\
\hline
\end{tabular}

Anmerkungen. Alle Analysen wurden mit HLM 5 (Raudenbush et al., 2000) durchgeführt.

a Schätzung der Varianzaufklärung ergab einen geringfügig negativen Wert (vgl. Fliesstext).

${ }^{* * *} p<.001{ }^{* *} p<.01 *^{*} p<.05$.

bReferenzgruppe: Freiburg

\section{Diskussion}

Die vorliegende Studie untersuchte für zentrale Indikatoren des Hausaufgabenverhaltens, wie stark sich einzelne Klassen und Schulen unterscheiden und ob diese Unterschiede durch strukturelle Parameter wie Klassengrösse und Zugehörigkeit zu einem bestimmten Kanton erklärt werden können. Im Einklang mit unseren Annahmen zeigten die Befunde, dass durchaus bedeutsame Unterschiede zwischen Klassen und Schulen vorliegen, die nur teilweise durch die überprüften strukturellen Aggregatvariablen verursacht wurden. Wir diskutieren im Folgenden wichtige Befunde und Implikationen unserer Studie. 


\section{Multidimensionalität, Mehrebenenperspektive und Fremd- sprachenunterricht}

$\mathrm{Zu}$ den zentralen Kritikpunkten an einem grossen Teil der Hausaufgabenforschung aus dem 20. Jahrhundert gehören die fehlende theoretische Einbindung und mangelhafte methodische Anlage vieler Studien (Cooper, 1989; Trautwein \& Köller, 2003a). Einer der Kritikpunkte betraf die Hausaufgabenzeit-Variable, deren inhaltliche Validität und Messgenauigkeit bezweifelt wurde. In der vorliegenden Studie haben wir deshalb neben der Hausaufgabenzeit weitere Variablen eingesetzt, die sich im Rahmen der Selbstregulationstheorie verorten lassen (Hausaufgabenengagement, Lernstrategien). Damit folgten wir dem Prinzip, dass das Hausaufgabenverhalten multidimensional zu konzipieren ist. Ein weiteres wichtiges Merkmal der Studie ist, dass sie eine der wenigen Arbeiten ist (siehe Haag \& Mischo, 2002), in der explizit Hausaufgaben im Fremdsprachenunterricht untersucht und diesbezügliche eine fachspezifische Konzeptualisierung der Hausaufgabenvariablen vorgenommen wurde.

Zweifellos das wichtigste Merkmal der vorliegenden Arbeit ist jedoch die Anwendung eines Mehrebenenmodells, das es erlaubt, Individualeffekte bei der Hausaufgabenerledigung von Klassen- und Schuleffekten zu trennen. Bei acht der 14 untersuchten Variablen waren mehr als 10 Prozent der Varianz nicht auf Individualebene lokalisiert. Dies ist durchaus ein beachtlicher Wert. Es lässt sich demnach konstatieren, dass sich Schüler aus einzelnen Klassen bzw. Schulen in ihrem Hausaufgabenverhalten durchaus bedeutsam unterscheiden, wobei je nach gewähltem Indikator das Ausmass der Unterschiede differiert.

\section{Schul-, Klassen- und Individualeffekte}

Die Anlage der Studie, bei der innerhalb der Schulen mehrere Klassen einbezogen wurden, machte es möglich, zwischen Schul- und Klasseneffekten zu unterscheiden und die strukturellen Prädiktorvariablen auf der jeweils angemessenen Ebene zu spezifizieren. Die Schulebene band zwischen einem und 17 Prozent der Varianz. Dabei fällt auf, dass auf der Schulebene die Prädiktorvariablen einen grossen Anteil der Varianz erklären konnten. Besonders systematisch ist der Effekt der Kantonszugehörigkeit. Im Vergleich zu Schülerinnen und Schülern aus Freiburg berichteten jene aus dem Kanton Wallis insgesamt über längere und regelmässigere Arbeitszeiten im Fach Französisch und eine systematischere Aufarbeitung von unklarem Stoff, jedoch über eine weniger aktive Nutzung des Französischen im Alltagsleben. Die Vermutung liegt nahe, dass vorgegebene Opportunitätsstrukturen zu diesen Befunden beitragen: So ist beispielsweise im Kanton Freiburg die überwiegend französischsprachige Hauptstadt Freiburg das Zentrum in Hinblick auf Kultur und Einkauf, während im Wallis die deutschsprachigen Kleinstädte Brig und Visp diese Funktion übernehmen. Zudem lässt sich spekulieren, dass bewusste pädagogische Zielsetzungen (z.B. die Vorgabe, regelmässig Hausaufaufgaben zu erteilen) und/oder spezifische Traditionen des Schüler/Lehrer-Verhältnisses einen Einfluss haben. Beispielsweise könnte es sein, 
dass im Wallis mit seiner vergleichsweise homogenen, katholisch geprägten Sozialstruktur autoritative Elemente deutlicher hervortreten als in den anderen beiden Kantonen.

Die Klassenebene band zwischen einem und 14 Prozent der Varianz. Die Varianzaufklärung durch die Prädiktorvariablen Klassengrösse und Kurszugehörigkeit war eher moderat. Uns scheint die Beobachtung besonders bedeutsam, dass gerade bei der Sorgfalt sowie bei der Regelmässigkeit der Hausaufgabenbearbeitung (Saisonarbeiter-Skala) - also zwei Kernvariablen des selbstregulierten Lernens - auf Klassenebene kaum Varianz erklärt wurde, obwohl dort immerhin knapp neun bzw. sieben Prozent der Gesamtvarianz lokalisiert waren. In anderen Worten: Schülerinnen und Schüler unterschiedlicher Klassen bearbeiten ihre Hausaufgaben in der Tat unterschiedlich sorgfältig, aber dieses Verhalten kovariiert kaum mit der Klassengrösse oder dem Kursniveau. Vielmehr ist anzunehmen, dass das Lehrerhandeln - und hier vermutlich in besonderem Masse auch die Qualität der Hausaufgaben - einen signifikanten Einfluss auf die Hausaufgabenbearbeitung der Schülerinnen und Schüler hat. Es stellt damit eine besonders wichtige Aufgabe für die zukünftige Hausaufgabenforschung dar, theoretisch fundierte Modelle der Hausaufgabenqualität zu entwickeln und den Effekt von Hausaufgabenqualität auf die Hausaufgabenerledigung zu überprüfen.

Schliesslich ist der grosse Varianzanteil zu nennen, der auf Individualebene lokalisiert ist. In dem vorliegenden Beitrag wurde darauf verzichtet, Prädiktoren auf Individualebene zu berücksichtigen, die das unterschiedliche Hausaufgabenverhalten vorhersagen können. Unter Bezugnahme auf Trautwein und Köller (2003b) darf vermutet werden, dass einerseits motivationale Prädiktoren, andererseits jedoch auch das Vorwissen und die Begabung sowie der familiäre Hintergrund eine bedeutsame Rolle spielen dürften. Gerade für den Fremdsprachenunterricht gilt zudem, dass ausserschulische Opportunitätsstrukturen (z.B. zweisprachiges Familienumfeld) besonders relevant sind.

\section{Beschränkungen der Studie und Ausblick}

Hinzuweisen ist darauf, dass in der vorliegenden Studie trotz einer multidimensionalen Konzeptualisierung der Hausaufgaben natürlich nicht alle potentiell wichtigen Aspekte von Hausaufgabenvergabe und -erledigung berücksichtigt werden konnten. Insbesondere in Hinblick auf die Erfassung der Lernstrategien lässt sich überlegen, ob nicht in Folgestudien weitere und verbesserte Instrumente zum Einsatz kommen könnten (vgl. Baumert \& Köller, 1996). Dies mag einerseits metakognitive Strategien betreffen, andererseits aber auch die Art und Weise des Vokabellernens.

Um einer Überinterpretation der signifikanten wie auch nicht-signifikanten Effekte auf Schulebene vorzubeugen, soll zudem ausdrücklich auf die kleine Zahl von untersuchten Einheiten auf der Schulebene verwiesen werden. Obwohl die Gesamtzahl der untersuchten Schulen es gerade noch als gerechtfertigt erscheinen liess, ein Drei-Ebenen-Modell zu spezifizieren, erfolgt die Schätzung 
des Einflusses der Prädiktorvariablen in einem solchen Falle mit einer geringen Teststärke.

Die bedeutsamen Varianzanteile auf Klassenebene (d.h. die Unterschiede zwischen den einzelnen Klassen), die für verschiedene Variablen nachgewiesen wurden, deuten auf Effekte der Lehrpersonen hin. Als einschränkende Bemerkung muss hierbei jedoch festgehalten werden, dass bedeutsame Varianzanteile auf Klassenebene auch von Kompositionseffekten (Schüler mit bestimmten Merkmalen ballen sich in bestimmten Klassen) herrühren können. Mit Hilfe von längsschnittlichen Analysen lässt sich dieses Problem entschärfen.

Die vorliegende Studie hat für wichtige Hausaufgabenvariablen die Varianzverteilung sowie den Effekt struktureller Prädiktoren berichtet. Allerdings wurden diese Hausaufgabenvariablen bislang nicht mit Leistung bzw. Leistungszuwachs in Verbindung gebracht. Selbstverständlich ist dies ein Schritt, der in dem Hausaufgabenprojekt in nächster Zukunft erfolgen soll. Dabei sollte nach Möglichkeit - wie in der vorliegenden Studie geplant - ein längsschnittliches Design verwendet werden, um einerseits die Effekte der Hausaufgabenvergabe und -erledigung auf die Leistungsentwicklung, andererseits aber auch die Effekte der Leistung auf die Hausaufgabenvergabe überprüfen zu können. In dem vorliegenden Forschungsprojekt soll dabei in künftigen, noch vorzunehmenden Analysen auch explizit die Hausaufgabenqualität einbezogen werden, die aus mehreren Perspektiven (Schülersicht, Selbstsicht der Lehrkräfte sowie Beurteilung durch fachdidaktisch geschulte Dritte) eingeschätzt wird.

\section{Anmerkungen}

Die vorliegende Studie wurde mit Mitteln des Schweizerischen Nationalfonds zur Förderung der wissenschaftlichen Forschung (DORE Projekt Nr. 13DPD3-108054/1) unterstützt.

Die Autoren danken Monika Oppong und Karin Buchs für die Hilfe bei der Datenerhebung und -aufbereitung sowie Jürgen Baumert für wertvolle Hinweise zu einer früheren Version des Manuskripts. Korrespondenz richten Sie bitte an Inge Schndyer (inge.schnyder@unifr.ch) oder Ulrich Trautwein (trautwein@mpib-berlin.mpg.de).

1 Obwohl der Begriff Hausaufgaben klassischer Weise enger gefasst wird (als «vom Lehrer erteilte Aufgaben»), wird dieser Begriff bei Trautwein und Köller (2003b) sowie in der vorliegenden Arbeit synonym mit dem breiteren Begriff des «häuslichen Lernens» verwendet.

2 In Bezug auf die Varianzaufklärung durch Prädiktoren ist ein Warnhinweis angebracht. Wenn in Mehrebenenanalysen auf einer Ebene nur sehr wenig Varianz lokalisiert ist (wie hier auf der Schulebene), sind die HLM-Schätzungen des Modells wenig robust, und es kann bei den Schätzungen der Varianzaufklärung auf der jeweiligen Ebene durch den Einbezug von Prädiktoren bekannter Weise zu fehlerbehafteten Schätzungen kommen (vgl. Hox, 2002). 


\section{Literaturverzeichnis}

Anderman, E. M. (2002). School effects on psychological outcomes during adolescence. Journal of Educational Psychology, 94, 646-648.

Artelt, C. (2000). Strategisches Lernen. Münster: Waxmann.

Baumert, J. \& Köller, O. (1996). Lernstrategien und schulische Leistungen. In J. Möller \& O. Köller (Hrsg.), Emotionen, Kognitionen und Schulleistung (S. 137-154). Weinheim: PVU.

Carroll, J. B. (1963). A model of school learning. Teachers' College Record, 64, 723-733.

Cooper, H. (1989). Homework. White Plains, NY: Longman.

Cooper, H., Robinson, J. C. \& Patall, E. A. (2006). Does homework improve academic achievement? A synthesis of research, 1987-2003. Review of Educational Research, 76, 1-62.

Corno, L. (2000). Looking at homework differently. The Elementary School Journal, 100, 529548.

De Jong, R., Westerhof, K. J. \& Creemers, B. P. M. (2000). Homework and student math achievement in junior high schools. Educational Research and Evaluation, 6, 130-157.

Eccles, J. S. (1983). Expectancies, values, and academic choice: Origins and changes. In J. Spence (Ed.), Achievement and achievement motivation (pp. 87-134). San Francisco: W. H. Freeman.

Farrow, S., Tymms, P. \& Henderson, B. (1999). Homework and attainment in primary schools. British Educational Research Journal, 25, 323-341.

Haag, L. \& Mischo, C. (2002). Saisonarbeiter in der Schule - einem Phänomen auf der Spur. Zeitschrift für Pädagogische Psychologie, 16, 109-115.

Hox, J. J. (2002). Multilevel analysis: Techniques and applications. Mahwah, NJ: Lawrence Erlbaum Associates.

Jamieson-Noel, D. \& Winne, P. H. (2003). Comparing self-reports to traces of studying behavior as representations of students' studying and achievement. Zeitschrift für Pädagogische Psychologie, 17, 159-171.

Lockl, K. \& Schneider, W. (2003). Metakognitive Überwachungs- und Selbstkontrollprozesse bei der Lernzeiteinteilung von Kindern. Zeitschrift für Pädagogische Psychologie, 17, 173-183.

Muhlenbruck, L., Cooper, H., Nye, B. \& Lindsay, J. J. (2000). Homework and achievement: Explaining the different strengths of relation at the elementary and secondary school levels. Social Psychology of Education, 3, 295-317.

Oxford, R. L. (1990). Language Learning Strategies: What Every Teacher Should Know. Boston: Heinle \& Heinle.

Raudenbush, S. W. \& Bryk, A. S. (2002). Hierarchical linear models (2nd ed.). Thousand Oaks: Sage.

Raudenbush, S. W., Bryk, A. S., Cheong, Y. \& Congdon, R. T. (2000). HLM 5: Hierarchical linear modeling. Chicago: Scientific Software International.

Schnyder, I., Niggli, A., Cathomas, R., Trautwein, U. \& Lüdtke, O. (2006). Wer lange lernt, lernt noch lange nicht viel mehr: Korrelate der Hausaufgabenzeit im Fach Französisch und Effekte auf die Leistungsentwicklung. Psychologie in Erziehung und Unterricht, 53, 107-121.

Stanat, P. \& Kunter, M. (2001). Kooperation und Kommunikation. In J. Baumert, E. Klieme, M. Neubrand, M. Prenzel, U. Schiefele, W. Schneider, et al. (Eds.), PISA 2000: Basiskompetenzen von Schülerinnen und Schülern im internationalen Vergleich (pp. 299-322). Opladen: Leske + Budrich.

Trautwein, U. \& Köller, O. (2003a). The relationship between homework and achievement still much of a mystery. Educational Psychology Review, 15, 115-145.

Trautwein, U. \& Köller, O. (2003b). Was lange währt, wird nicht immer gut: Zur Rolle selbstregulativer Strategien bei der Hausaufgabenerledigung. Zeitschrift für Pädagogische Psychologie, 17, 199-209.

Trautwein, U., Köller, O., Schmitz, B. \& Baumert, J. (2002). Do homework assignments enhance achievement? A multilevel analysis of 7 th grade mathematics. Contemporary Educational Psychology, 27, 26-50. 
Trautwein, U. \& Lüdtke, O. (2004). Aspekte von Wissenschaftspropädeutik und Studierfähigkeit. In O. Köller, R. Watermann, U. Trautwein \& O. Lüdtke (Hrsg.), Wege zur Hochschulreife in Baden-Württemberg. TOSCA - Eine Untersuchung an allgemein bildenden und beruflichen Gymnasien (S. 327-366). Opladen: Leske + Budrich.

Wagner, P. \& Spiel, C. (2002a). Hausaufgabenforschung - ein Plädoyer für eine stärkere theoretische Verankerung. Empirische Pädagogik, 16, 275-284.

Wagner, P. \& Spiel, C. (2002b). Zeitinvestment und Lerneffektivität: Eine Analyse in Hauptschule und Gymnasium hinsichtlich Persönlichkeitsvariablen, Arbeitshaltung und Bedingungsfaktoren. Empirische Pädagogik, 16, 357-382.

Walberg, H. J. \& Paschal, R. A. (1995). Homework. In L. W. Anderson (Ed.), International encyclopaedia of teaching and teacher education (pp. 268-271). Oxford: Elsevier.

Schlagworte: Hausaufgaben, Fremdsprache, Schulleistung, Mehrebenenanalysen

\section{L'attitude des élèves à l'égard des devoirs change-t-elle d'une classe à l'autre? Résultats d'une analyse multiniveau dans la branche "français comme langue étrangère "}

\section{Résumé}

On attribue à la distribution de devoirs un apport significatif à la performance scolaire. Pourtant, les différences constatées dans l'attitude moyenne des classes à l'égard des devoirs restent jusqu'à présent en grande partie inexpliquées de même que l'influence de facteurs plus généraux sur l'accomplissement des devoirs, tels que le type de la classe, sa taille ou l'appartenance à un canton

La présente enquête a été menée auprès de 1312 élèves de huitième année appartenant à 73 classes répartites dans trois cantons, et dont l'allemand est la langue utilisée pour l'enseignement. Pour présumer de la motivation et du comportement des élèves lors de l'exécution des devoirs, des analyses multiniveau ont été menées.

Ces analyses montrent que des facteurs dépendant de la classe et de l'établissement scolaire influencent considérablement l'apprentissage des élèves à la maison. En même temps, il apparaît que la seule prise en considération d'aspects structurels tels que la taille ou le type de la classe n'est pas suffisante. La recherche sur la qualité des devoirs demande à être approfondie.

Mots clés: Devoirs, langue étrangère, performance scolaire, analyse multiniveau 


\section{L'atteggiamento degli allievi di fronte ai compiti cambia da una classe all'altra? Risultati da un'analisi a più livelli in francese $L 2$}

\section{Riassunto}

Ai compiti si attribuisce un contributo significativo ai risultati scolastici. Finora non si è però mai chiarito in che misura gli atteggiamenti verso i compiti variano mediamente da una classe all'altra e quale incidenza abbiano fattori aggregati (ad es. livello dei corsi, dimensioni della classe, provenienza cantonale). Lo studio analizza 1312 allievi dell'ottavo anno in 73 classi di tre Cantoni svizzero tedeschi, classi dove la lingua d'insegnamento è il tedesco. I fattori presi in esame sono la motivazione e le modalità di svolgimento dei compiti da parte degli allievi. Gli esiti mostrano come questi fattori vengano influenzati in modo significativo dalle variabili attinenti alla classe e alla scuola. Al tempo stesso si nota che la sola considerazione di variabili strutturali come la dimensione della classe o il livello del corso non sono sufficienti. Si conclude suggerendo la necessità di ulteriori approfondimenti.

Parole chiave: compiti, lingua seconda, prestazione scolastica

\section{Are there any differences in homework behavior in students from different classrooms? Results from a multilevel analysis in French as a second language}

\section{Summary}

Students' achievement gains have been linked to homework assignments (teacher level) and students' conscientious homework behavior (student level). However, there are no empirical studies that empirically investigated the differences in homework behavior among students in different classes and the impact of structural features (e.g., course level, class size, region) on students' homework behavior. In the present study with 1,312 eight graders from 73 German speaking classes from three regions in Switzerland, multilevel modeling was used to predict students' homework motivation and behavior. The analyses indicate that school-level and course-level factors significantly impact on students' homework behavior. However, the data indicate that it is not sufficient to take structural predictors such as class size and course level into account. Therefore, studies focusing on homework quality are called for.

Key words: Homework, second language acquisition, scholastic achievement, multilevel modeling 
